\title{
High-flow nasal cannula oxygen therapy in idiopathic pulmonary fibrosis patients with respiratory failure
}

\author{
Ji-Hoon Lee ${ }^{1}$, Chae-Man Lim², Younsuck Koh' ${ }^{2}$, Sang-Bum Hong ${ }^{2}$, Jin-Woo Song ${ }^{2}$, Jin Won Huh ${ }^{2}$ \\ ${ }^{1}$ Department of Pulmonary and Critical Care Medicine, Dongsuwon General Hospital, Suwon, Republic of Korea; ${ }^{2}$ Department of Pulmonary and \\ Critical Care Medicine, Asan Medical Center, College of Medicine, University of Ulsan, Seoul, Republic of Korea \\ Contributions: (I) Conception and design: JW Huh; (II) Administrative support: CM Lim, Y Koh, SB Hong; (III) Provision of study materials or \\ patients: JW Song, JW Huh; (IV) Collection and assembly of data: JH Lee; (V) Data analysis and interpretation: JH Lee; (VI) Manuscript writing: \\ All authors; (VII) Final approval of manuscript: All authors. \\ Correspondence to: Jin Won Huh, MD, PhD. Department of Pulmonary and Critical Care Medicine, Asan Medical Centre, University of Ulsan \\ College of Medicine, 88, Olympic-ro 43-gil, Songpa-gu, Seoul 05505, Republic of Korea. Email: jwhuh@amc.seoul.kr.
}

\begin{abstract}
Background: High-flow nasal cannula (HFNC) oxygen therapy is widely applied in idiopathic pulmonary fibrosis (IPF) patients with acute respiratory failure (ARF); however, its advantages over mechanical ventilation $(\mathrm{MV})$ remain unclear. We aimed to compare the clinical outcomes of HFNC oxygen therapy and MV in IPF patients with respiratory failure.

Methods: A retrospective descriptive study of patients with IPF admitted between January 2015 and December 2017 who underwent HFNC oxygen therapy or MV during hospitalization was conducted. The primary outcome was the comparison of in-hospital mortality among HFNC only group, MV with prior HFNC group, and MV only group.

Results: A total of 61 patients with IPF and ARF were included in the current study. Forty-five patients received HFNC oxygen therapy without endotracheal intubation and 16 received MV. The overall hospital mortality rate was $59.0 \%$, of which $53.3 \%$ was for HFNC oxygen therapy and $55.6 \%$ (5/9) for MV only group $(\mathrm{P}=1.000)$. Although no significant difference in the mortality rate was observed among three groups, that of MV with prior HFNC oxygen therapy $(n=7)$ was $100 \%(P=0.064)$. Additionally, the HFNC oxygen therapy group showed shorter length of hospital and ICU stay than the MV group $(\mathrm{P}<0.001)$.

Conclusions: Patients with IPF and ARF who received MV with prior HFNC oxygen therapy showed increased mortality rate than those who received HFNC only oxygen therapy or MV. Considering the complication rate of $\mathrm{MV}$, need for lung transplantation, and the will to undergo end-of-life care, a proper transition from HFNC oxygen therapy to MV should be planned cautiously.
\end{abstract}

Keywords: Idiopathic pulmonary fibrosis (IPF); high flow nasal cannula (HFNC); mechanical ventilation (MV)

Submitted Aug 07, 2019. Accepted for publication Nov 27, 2019.

doi: $10.21037 /$ jtd.2019.12.48

View this article at: http://dx.doi.org/10.21037/jtd.2019.12.48

\section{Introduction}

Idiopathic pulmonary fibrosis (IPF) is a serious disease that causes respiratory failure by progressive lung fibrosis. Studies have reported an increase in the incidence of IPF and the resultant deaths $(1,2)$. In recent years, although nintedanib and pirfenidone have been shown to be effective disease-modifying agents, their beneficial effect on the prognosis of patients with IPF has been limited. Lung transplantation is the only definitive therapy in pharmacologically refractory IPF; however, only a small proportion of patients undergo the procedure due to insufficient supply of donor organs, technical challenges associated with the transplantation, or peri-operative conditioning (3). In the United States, the median period of survival of patients with IPF aged 65 years and older is only 


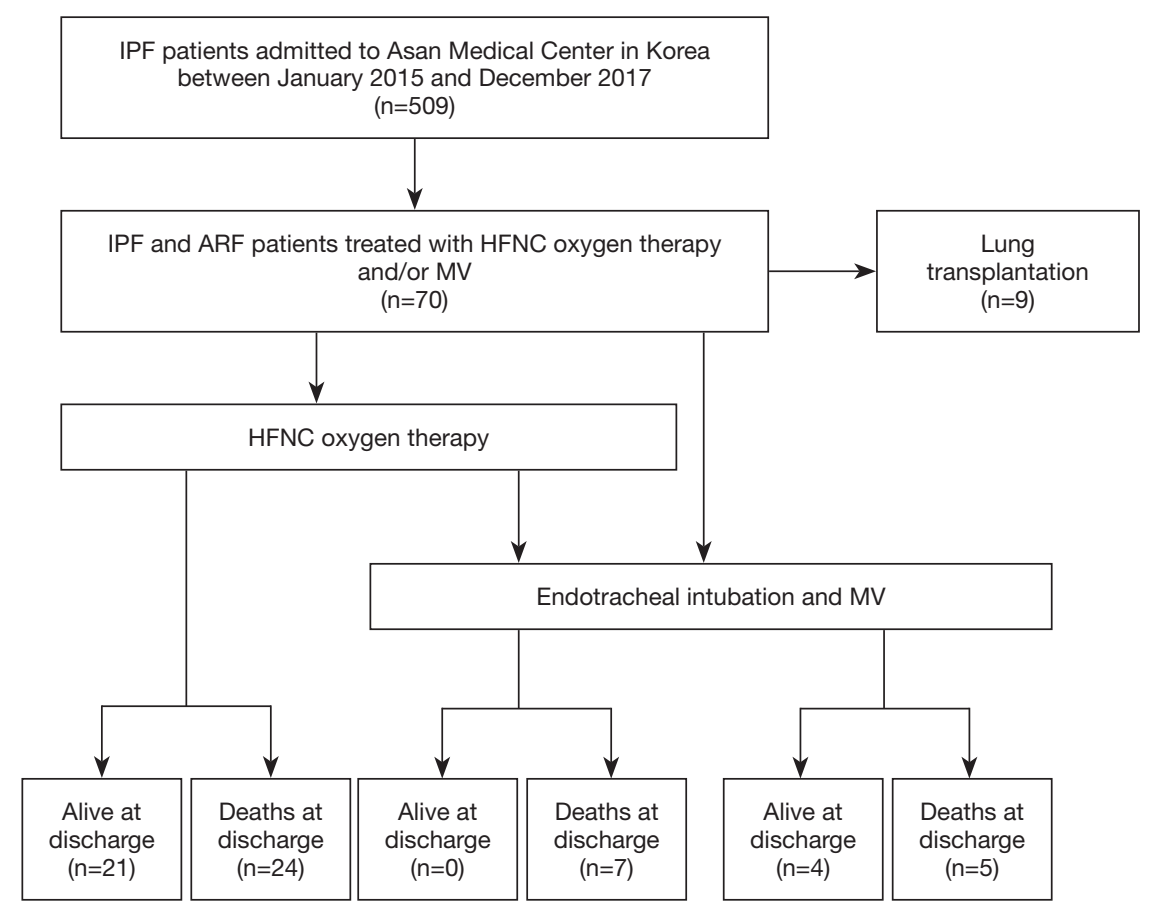

Figure 1 Distribution of the study population according to therapy and outcome. IPF, idiopathic pulmonary fibrosis; ARF, acute respiratory failure; HFNC, High-flow nasal cannula; MV, mechanical ventilation.

3.8 years (4). In particular, the prognosis is extremely poor in IPF patients experiencing acute respiratory failure (ARF), the in-hospital mortality reaching almost $85 \%$. Moreover, mechanical ventilation (MV) does not have a significant therapeutic effect on the survival, unlike in ARF caused by other etiologies (5). Impaired gas exchange and reduced pulmonary compliance lead to high peak airway pressure, tidal volume, and fraction of inspired oxygen $\left(\mathrm{FiO}_{2}\right)$. These traumatic components could accelerate progression of the disease (6).

High-flow nasal cannula (HFNC) oxygen therapy is a technique that can deliver a $\mathrm{FiO}_{2}$ up to 1.0 by supplying warm, humidified oxygen through a nasal cannula. Recently, various attempts have been made to apply HFNC oxygen therapy to patients with ARF. Although the technique does not reduce the need for endotracheal intubation or mortality compared to conventional oxygen therapy or noninvasive ventilation (NIV), it has advantages such as patient comfort and tolerability (7). We reported that delayed intubation in the late HFNC oxygen therapy failure group increases the mortality rate (8). Because we included various etiologies of respiratory failure for analysis in this study, the outcome of HFNC oxygen therapy in IPF patients with the high mortality rate associated with MV may be different (9).
The current study compared the clinical outcomes of HFNC oxygen therapy and MV in IPF patients admitted due to ARF.

\section{Methods}

\section{Patients and study design}

The institutional review board of Asan Medical Center (IRB No. 2019-0222) approved the current study. Informed consent was waived owing to the retrospective design of the study based on medical records.

This retrospective descriptive study was conducted on patients with IPF who were admitted to the Asan Medical Center, Seoul, Korea, from January 2015 to December 2017 and underwent HFNC oxygen therapy or MV during hospitalization (Figure 1). Patients were divided into three groups (HFNC only group, MV with prior HFNC group and MV only group). The study subjects were limited to those who had an established diagnosis of IPF according to the statement of the American Thoracic Society (ATS)/ European Respiratory Society (ERS)/Japanese Respiratory Society (JRS)/Latin American Thoracic Association (ALAT) (10), and who were followed up at this hospital. 


\section{Data collection}

The demographic factors and clinical parameters of the patients were reviewed from the electronic medical records. The results of the most recent (within six months) spirometry and diffusing capacity of the lung for carbon monoxide (DLCO) were retrieved to assess the severity of IPF before admission and the GAP index (11) and du Bois score (12) were calculated based on this information. Status of the patients at the time of admission was confirmed by vital signs and arterial blood gas analysis (ABGA).

\section{Study outcomes}

The primary outcome measure was in-hospital mortality, which was compared among HFNC only group, MV with prior HFNC group, and MV only group. Other prespecified outcome measures were length of hospital stay, length of intensive care unit (ICU) stay, and the proportion of do-not-resuscitate (DNR) consent status. The vital signs were evaluated in patients who underwent HFNC oxygen therapy before the initiation of treatment and after 8 hours of treatment to indirectly assess respiratory distress. Ventilator-associated complications were reviewed in patients who underwent MV. Possible ventilator-associated pneumonia (PVAP) was defined based on the ventilatorassociated event (VAE) surveillance algorithm definition by the Centers for Disease Control and Prevention (CDC) National Health Safety Network (13).

\section{Statistical analyses}

The results are represented as mean values and standard deviations (SD) or median and interquartile range (IQR) for continuous variables and numbers and percentages for categorical data. Group comparisons were based on the Fisher's exact test or $\chi^{2}$ test for categorical data, and Kruskal-Wallis test for continuous data. All statistical results were evaluated using SPSS for Windows (Version 21.0, IBM Corporation, Chicago, IL, USA).

\section{Results}

\section{Patient characteristics}

A total of 61 patients with IPF and ARF were included in the current study. There were a higher number of male patients $(n=48,78.7 \%)$, and the mean age was 70.8 years. No significant difference was observed among three groups, except the baseline history of coronary artery disease. Most patients had advanced IPF (Table 1). GAP index stage III was the most common and the mean du Bois score was 42.8. The vital signs and ABGA results of the patients were not significantly different between the groups at the time of admission (Table 2).

\section{Clinical course and outcome}

With the worsening of oxygenation, 45 patients received HFNC oxygen therapy without endotracheal intubation and 16 patients underwent MV. None of the subjects applied NIV during their hospital stay. Seven patients who received MV underwent endotracheal intubation for clinical progression such as hypoxemia and respiratory distress during HFNC oxygen therapy and nine patients received MV without prior application of HFNC oxygen therapy. In the HFNC only group, the average time from the admission to the appliance was 3.0 days and the PF ratio was $155.6 \pm 65.2 \mathrm{mmHg}$. The initial settings of HFNC oxygen therapy were a median flow rate of $35 \mathrm{~L} / \mathrm{min}$ (IQR 35 to 40 ) and $\mathrm{FiO}_{2}$ of 0.60 (IQR 0.50 to 0.80 ). Respiratory and pulse rate decreased at eight hours after application of HFNC oxygen therapy from 28.9 to 24.5 breaths/min and from 103.9 to 90.5 beats/min, respectively (Table 3).

The PF ratio before intubation in the MV only group was $143.4 \pm 75.5 \mathrm{mmHg}$. In the MV with prior HFNC oxygen therapy group, the average time from application of HFNC oxygen therapy to MV was 5 (3-5.5) days and the PF ratio was $120.4 \pm 55.9 \mathrm{mmHg}$ at intubation. In patients who underwent MV, PVAP occurred in five of 16 patients (31.2\%), and barotrauma (e.g., pneumomediastinum or pneumothorax) occurred in two patients (12.5\%) during the procedure.

The hospital mortality rate of the study subjects was $59.0 \%$ (36/61), 53.3\% (24/45) of which was for HFNC oxygen therapy only group and $55.6 \%(5 / 9)$ for MV only group ( $\mathrm{P}=1.000)$. Although no difference in mortality was observed among the three groups, the mortality rate of patients who underwent $M V$ with prior HFNC oxygen therapy was $100 \%(\mathrm{P}=0.064)$. The $87.5 \%$ (21/24) of non-survivors in the HFNC only group was with their family during the end of life. Significant difference was observed between the groups in terms of the lengths of hospital and ICU stays $(\mathrm{P}<0.001$ each) (Table 4).

\section{Discussion}

The current study retrospectively reviewed the clinical outcomes of advanced IPF patients with respiratory failure who required HFNC oxygen therapy or MV. The 
Table 1 Baseline characteristics of study patients

\begin{tabular}{|c|c|c|c|c|}
\hline Characteristics & HFNC only group $(n=45)$ & MV with prior HFNC $(n=7)$ & MV only group $(n=9)$ & $P$ value ${ }^{\dagger}$ \\
\hline Age, years & $71.0 \pm 7.6$ & $69.9 \pm 8.8$ & $70.4 \pm 9.1$ & 0.974 \\
\hline $\mathrm{BMI}\left(\mathrm{kg} / \mathrm{m}^{2}\right)$ & $23.8 \pm 3.2$ & $25.3 \pm 4.0$ & $22.8 \pm 2.9$ & 0.523 \\
\hline TLC, \% predicted* & $43.0 \pm 22.7$ & $49.6 \pm 9.9$ & $50.1 \pm 21.1$ & 0.602 \\
\hline $\mathrm{FEV}_{1}, \%$ predicted $^{\star}$ & $56.6 \pm 12.0$ & $52.1 \pm 11.4$ & $63.7 \pm 13.2$ & 0.208 \\
\hline $\mathrm{D}_{\mathrm{Lco}}, \%$ predicted ${ }^{*}$ & $23.6 \pm 14.5$ & $24.3 \pm 6.1$ & $23.6 \pm 22.7$ & 0.981 \\
\hline Home oxygen therapy, n (\%) & $34(75.6)$ & $4(57.1)$ & $6(66.7)$ & 0.555 \\
\hline IPF treatment, $\mathrm{n}(\%)$ & & & & 0.232 \\
\hline Glucocorticoids & $21(46.7)$ & $3(42.9)$ & $3(33.3)$ & \\
\hline Other & $2(4.4)$ & $0(0.0)$ & $2(22.2)$ & \\
\hline Treatment interruptions & $8(17.8)$ & $1(14.3)$ & $4(44.4)$ & \\
\hline GAP index stage, $n(\%)$ & & & & 0.750 \\
\hline II & $13(28.9)$ & $3(42.9)$ & $3(33.3)$ & \\
\hline III & $32(71.1)$ & $4(57.1)$ & $6(66.7)$ & \\
\hline du Bois score & $42.4 \pm 12.3$ & $50.9 \pm 10.3$ & $41.6 \pm 16.7$ & 0.261 \\
\hline \multicolumn{5}{|l|}{ Underlying disease, n (\%) } \\
\hline Pulmonary hypertension & $28(62.2)$ & $3(42.9)$ & $5(55.6)$ & 0.609 \\
\hline
\end{tabular}

HFNC, high flow nasal cannula; BMI, body mass index; TLC, total lung capacity; FVC, forced vital capacity; $D_{\text {Lco, }}$ carbon monoxide diffusing capacity; GAP, gender, age, and physiology. ${ }^{*}$ Spirometry and $D_{\text {LCo }}$ values are based on the most recent values within 6 months before admission. ${ }^{\dagger} \mathrm{P}$ value $<0.05$ among HFNC group, MV with prior HFNC, and MV only group.

proportion of HFNC oxygen therapy was higher than MV in IPF patients with ARF. Most patients in the HFNC oxygen therapy group stayed with their family in the general ward, and non-survivors of this group received end-of-life care under DNR state. Although no significant difference in the in-hospital mortality rate was observed between the HFNC oxygen therapy and MV groups, the mortality rate of patients in the HFNC oxygen therapy failure group who were transitioned to MV was extremely high.
Respiratory failure in patients with IPF patients carries a miserable outcome, and no effective treatment is present to reverse progression of the condition (14). More than half of the patients in the current study died in the hospital, regardless of application of HFNC oxygen therapy or MV. In addition to not being able to reverse the course of the disease, the choice of MV could cause patient discomfort, increased requirement for sedation and ventilator-associated pneumonia. Particularly, the lung is vulnerable to the stress 
Table 2 Physiologic and laboratory data of patients at hospital admission

\begin{tabular}{|c|c|c|c|c|}
\hline Parameters & HFNC only group $(n=45)$ & MV with prior HFNC $(n=7)$ & MV only group $(n=9)$ & $P$ value ${ }^{\dagger}$ \\
\hline Diastolic blood pressure $(\mathrm{mmHg})$ & $76.7 \pm 15.2$ & $75.9 \pm 15.4$ & $68.9 \pm 20.1$ & 0.512 \\
\hline Pulse rate (beats/min) & $109.7 \pm 21.1$ & $113.1 \pm 20.3$ & $120.0 \pm 28.7$ & 0.819 \\
\hline Respiratory rate (breaths/min) & $30.1 \pm 8.2$ & $22.6 \pm 3.2$ & $28.9 \pm 4.8$ & 0.010 \\
\hline Arterial $\mathrm{pH}$ & $7.45 \pm 0.06$ & $7.45 \pm 0.06$ & $7.39 \pm 0.06$ & 0.056 \\
\hline $\mathrm{PaCO}_{2}(\mathrm{mmHg})$ & $37.1 \pm 6.6$ & $39.3 \pm 12.0$ & $42.4 \pm 7.1$ & 0.130 \\
\hline $\mathrm{PaO}_{2}(\mathrm{mmHg})$ & $82.9 \pm 47.7$ & $87.9 \pm 44.0$ & $97.6 \pm 42.0$ & 0.366 \\
\hline Bicarbonate $(\mathrm{mmHg})$ & $25.8 \pm 4.1$ & $26.9 \pm 6.2$ & $25.8 \pm 3.3$ & 0.910 \\
\hline
\end{tabular}

${ }^{\dagger} \mathrm{P}$ value $<0.05$ among HFNC group, MV with prior HFNC, and MV only group. HFNC, high flow nasal cannula; MV, mechanical ventilation.

Table 3 Changes in vital sign after application of HFNC

\begin{tabular}{|c|c|c|c|}
\hline Parameters & Pre-HFNC application & Post-HFNC application & $P$ value \\
\hline Diastolic blood pressure $(\mathrm{mmHg})$ & $74.1 \pm 13.5$ & $74.3 \pm 11.8$ & 0.785 \\
\hline Pulse rate (beats/min) & $103.9 \pm 17.1$ & $90.5 \pm 16.7$ & $<0.001$ \\
\hline Respiratory rate (breaths/min) & $28.9 \pm 6.0$ & $24.5 \pm 3.6$ & $<0.001$ \\
\hline
\end{tabular}

HFNC, high flow nasal cannula.

Table 4 Clinical outcomes

\begin{tabular}{|c|c|c|c|c|}
\hline Parameters & HFNC only group $(n=45)$ & MV with prior HFNC $(n=7)$ & MV only group $(n=9)$ & $P$ value ${ }^{\dagger}$ \\
\hline Deaths in ICU, n (\%) & $3(6.7)$ & $7(100.0)$ & $5(55.6)$ & $<0.001$ \\
\hline Length of hospital stay, days & $13(7-18)$ & $19(11-38.5)$ & $24(12-31)$ & 0.134 \\
\hline Length of ICU stay, days & $0(0-0)^{\ddagger}$ & $14(8-24.5)$ & $7(6-15)$ & $<0.001$ \\
\hline Death without DNR consent, n (\%) & $0(0.0)$ & $4(57.1)$ & $1(11.1)$ & 0.001 \\
\hline Time to death after DNR consent, days & $1(0-4)$ & $0(0-1)$ & $0(0-1)$ & 0.563 \\
\hline
\end{tabular}

HFNC, high flow nasal cannula; MV, mechanical ventilation; DNR, do-not-resuscitate. ${ }^{\dagger} \mathrm{P}$ value $<0.05$ among HFNC group, MV with prior HFNC, and MV only group. ${ }^{\ddagger}$ Only three died in ICU and the lengths of ICU stay were 1,3 and 3 days, respectively.

of MV due to progression of fibrosis. In the current study, complications related to MV occurred in many patients in the MV group. When considering the distinct characteristics of the disease, endotracheal intubation in acute respiratory failure in patients with advanced IPF is controversial.
Nevertheless, according to a recent report, approximately $10 \%$ of IPF patients continue to receive $\mathrm{MV}$, and is associated with increased admission cost and mortality (9).

HFNC can deliver a high concentration of humidified oxygen at a fraction of inspired oxygen of $1.0(100 \%)$. 
The high flow rates of the nasal cannula generate a low level of positive pressure in the upper airway and lighten the work of breathing by reducing the physiologic dead space (15). HFNC oxygen therapy has gained popularity and is routinely used for the treatment of hypoxemic respiratory failure. Synthetically, HFNC oxygen therapy has the advantages of convenience and patient comfort when compared to invasive MV. Owing to its advantages, an expansion in the scope of application of HFNC oxygen therapy is being observed, both before and after intubation and end-of-life care, in addition to acute respiratory failure. Peters et al. have proposed HFNC oxygen therapy as an effective respiratory support for patients with respiratory failure in do-not-intubate status (16). HFNC oxygen therapy demonstrated palliative benefit, such as improved patient oxygenation and decreased respiratory rate, and was well tolerated. Similarly, reduction in the respiratory frequency and pulse rate of patients was observed after HFNC oxygen therapy in the current study. These findings suggest that HFNC oxygen therapy may be an effective adjunctive therapy for IPF patients with respiratory failure. However, in the current study, higher mortality was observed following the application of $\mathrm{MV}$ in cases of failure of HFNC oxygen therapy compared with other studies. In a previous study, high rate of mortality was observed when intubation was delayed due to failure of HFNC oxygen therapy (8), and mechanical ventilation may not have an additional therapeutic benefit because the cause of respiratory failure is not well corrected in patients with IPF with a pre-existing fibrotic component. Ventilator support can be used only as a bridge to lung transplant in cases of failure of HFNC oxygen therapy (17).

Early integrated palliative care is essential for patients with advanced IPF due to limited life expectancy and the heavy burden of symptoms. Despite recommendations for palliative care, the majority of IPF patients continue to receive life-prolonging procedures such as non-invasive ventilation, and end-of-life decisions are often delayed (18). Decision to refrain from intubation usually depends on the assessment of futility of care, wishes of the patient, and high probability of poor quality of life in the future (19). In the current study, non-survivors in the MV group showed lower agreement on the DNR order, which was very late, i.e., average 2 days prior to death. This may be associated with the low awareness of end-of-life care in patients with advanced IPF. In addition, patients with IPF receiving MV are referred to palliative care in a minority of cases (20). Therefore, in instances of no further treatment plan such as lung transplantation, the decision to perform invasive ventilation should be made in a discrete manner. HFNC oxygen therapy is advantageous in patients with advanced IPF in that they can make end-of-life decisions on their own until the last minute of consciousness, as patients are able to communicate unlike endotracheal intubation. HFNC oxygen therapy also plays a role in palliative care because symptoms such as shortness of breath can be relieved, and consumption of food and drink is facilitated. Therefore, in cases of patients with advanced IPF who are hospitalized with acute respiratory failure, wherein active integrated palliative care and end-oflife decisions are required, HFNC oxygen therapy may be the most appropriate modality for respiratory support.

The current study has several limitations. First, a single center, retrospective study might be associated with selection bias of patients. The application of the HFNC oxygen therapy or MV was based on the clinical decision of the physician. Although the baseline characteristics such age, pulmonary functions, and severity were compared, the sicker patient might have included in the MV group. However, a randomized trial is difficult due to the poor outcome of $M V$ and routine application of HFNC oxygen therapy. Second, too few patients were included to analyze the statistical meaningful difference. Third, intubation was an inevitable step when patients and families disagreed for DNR despite the clinician recognizing the poor prognosis of the disease.

\section{Conclusions}

In the current retrospective study, the HFNC group showed shorter duration of stay in the hospital and ICU without difference in mortality. However, the application of MV in the HFNC oxygen therapy failure group showed extremely higher mortality. Considering the complications of $M V$ and the end-of-life care, the further study for the guideline of the transition to $M V$ are needed.

\section{Acknowledgments}

Funding: None.

\section{Footnote}

Conflicts of Interest: The authors have no conflicts of interest to declare.

Etbical Statement: The authors are accountable for all aspects of the work in ensuring that questions related 
to the accuracy or integrity of any part of the work are appropriately investigated and resolved. The institutional review board of Asan Medical Center (IRB No. 2019-0222) approved the current study. Informed consent was waived owing to the retrospective design of the study based on medical records.

Open Access Statement: This is an Open Access article distributed in accordance with the Creative Commons Attribution-NonCommercial-NoDerivs 4.0 International License (CC BY-NC-ND 4.0), which permits the noncommercial replication and distribution of the article with the strict proviso that no changes or edits are made and the original work is properly cited (including links to both the formal publication through the relevant DOI and the license). See: https://creativecommons.org/licenses/by-nc-nd/4.0/.

\section{References}

1. Hutchinson J, Fogarty A, Hubbard R, et al. Global incidence and mortality of idiopathic pulmonary fibrosis: a systematic review. Eur Respir J 2015;46:795-806.

2. Hutchinson JP, McKeever TM, Fogarty AW, et al. Increasing global mortality from idiopathic pulmonary fibrosis in the twenty-first century. Ann Am Thorac Soc 2014;11:1176-85.

3. Kistler KD, Nalysnyk L, Rotella P, et al. Lung transplantation in idiopathic pulmonary fibrosis: a systematic review of the literature. BMC Pulm Med 2014;14:139.

4. Raghu G, Chen SY, Yeh WS, et al. Idiopathic pulmonary fibrosis in US Medicare beneficiaries aged 65 years and older: incidence, prevalence, and survival, 2001-11. Lancet Respir Med 2014;2:566-72.

5. Stern JB, Mal H, Groussard O, et al. Prognosis of patients with advanced idiopathic pulmonary fibrosis requiring mechanical ventilation for acute respiratory failure. Chest 2001;120:213-9.

6. Anzueto A, Frutos-Vivar F, Esteban A, et al. Incidence, risk factors and outcome of barotrauma in mechanically ventilated patients. Intensive Care Med 2004;30:612-9.

7. Monro-Somerville T, Sim M, Ruddy J, et al. The Effect of High-Flow Nasal Cannula Oxygen Therapy on Mortality and Intubation Rate in Acute Respiratory Failure: A Systematic Review and Meta-Analysis. Crit Care Med 2017;45:e449-56.

8. Kang BJ, Koh Y, Lim CM, et al. Failure of high-flow nasal cannula therapy may delay intubation and increase mortality. Intensive Care Med 2015;41:623-32.

9. Mooney JJ, Raimundo K, Chang E, et al. Mechanical ventilation in idiopathic pulmonary fibrosis: a nationwide analysis of ventilator use, outcomes, and resource burden. BMC Pulm Med 2017;17:84.

10. Raghu G, Collard HR, Egan JJ, et al. An official ATS/ ERS/JRS/ALAT statement: idiopathic pulmonary fibrosis: evidence-based guidelines for diagnosis and management. Am J Respir Crit Care Med 2011;183:788-824.

11. Ley B, Ryerson CJ, Vittinghoff E, et al. A multidimensional index and staging system for idiopathic pulmonary fibrosis. Ann Intern Med 2012;156:684-91.

12. du Bois RM, Weycker D, Albera C, et al. Ascertainment of individual risk of mortality for patients with idiopathic pulmonary fibrosis. Am J Respir Crit Care Med 2011;184:459-66.

13. Magill SS, Klompas M, Balk R, et al. Developing a new, national approach to surveillance for ventilator-associated events: executive summary. Chest 2013;144:1448-52.

14. Blivet S, Philit F, Sab JM, et al. Outcome of patients with idiopathic pulmonary fibrosis admitted to the ICU for respiratory failure. Chest 2001;120:209-12.

15. Delorme M, Bouchard PA, Simon M, et al. Effects of High-Flow Nasal Cannula on the Work of Breathing in Patients Recovering From Acute Respiratory Failure. Crit Care Med 2017;45:1981-8.

16. Peters SG, Holets SR, Gay PC. High-flow nasal cannula therapy in do-not-intubate patients with hypoxemic respiratory distress. Respir Care 2013;58:597-600.

17. Weill D, Benden C, Corris PA, et al. A consensus document for the selection of lung transplant candidates: 2014--an update from the Pulmonary Transplantation Council of the International Society for Heart and Lung Transplantation. J Heart Lung Transplant 2015;34:1-15.

18. Rajala K, Lehto JT, Saarinen M, et al. End-of-life care of patients with idiopathic pulmonary fibrosis. BMC Palliat Care 2016;15:85.

19. Mallick S. Outcome of patients with idiopathic pulmonary fibrosis (IPF) ventilated in intensive care unit. Respir Med 2008;102:1355-9.

20. Rush B, Berger L, Anthony Celi L. Access to Palliative Care for Patients Undergoing Mechanical Ventilation With Idiopathic Pulmonary Fibrosis in the United States. Am J Hosp Palliat Care 2018;35:492-6.

Cite this article as: Lee $\mathrm{JH}$, Lim CM, Koh Y, Hong SB, Song JW, Huh JW. High-flow nasal cannula oxygen therapy in idiopathic pulmonary fibrosis patients with respiratory failure. J Thorac Dis 2020;12(3):966-972. doi: 10.21037/jtd.2019.12.48 